\title{
Aplicabilidad de los modelos NAM y DBM para estimar caudales en subcuencas alto andinas de Ecuador
}

\author{
A. Quichimbo ${ }^{1}$, R.F. Vázquez ${ }^{1}$, E. Samaniego ${ }^{2}$ \\ ${ }^{1}$ Grupo de Ciencias de la Tierra y del Ambiente, Dirección de Investigación (DIUC), Universidad de \\ Cuenca, Av. 12 de abril S/N, Cuenca, Ecuador. \\ ${ }^{2}$ Facultad de Ingeniería, Universidad de Cuenca, Av. 12 de abril S/N, Cuenca, Ecuador. \\ Autor para correspondencia: raulfvazquezz@yahoo.co.uk \\ Fecha de recepción: 1 de junio de 2013 - Fecha de aceptación: 2 de septiembre 2013
}

\section{RESUMEN}

El modelo Mecanicista Basado en Datos (DBM), método híbrido que puede emplearse para la predicción de caudales, y el Nedbor-Afstromnings Model (NAM), modelo hidrológico conceptual agregado, se utilizaron para estudiar la relación lluvia-escorrentía de subcuencas andinas de diverso tamaño, ubicadas en el sur del Ecuador. El presente artículo detalla el procedimiento seguido para la aplicación de ambas estructuras de modelización, con énfasis en la evaluación de su desempeño. El estudio reveló que el modelo DBM es el que describe de mejor manera el sistema precipitaciónescorrentía, representando las subcuencas modelizadas por medio de una estructura que comprende tres reservorios lineales.

Palabras clave: Modelización hidrológica, DBM (Data-Based Mechanistic), Función de Transferencia (TF), Parámetros Dependientes de Estado (SDP), Nedbor-Afstromnings Model (NAM), calibración.

\begin{abstract}
A Data-Based Mechanistic (DBM) model and the Nedbor-Afstromnings Model (NAM) were applied to simulate the rainfall-runoff relationship of two Andean basins, different in size, located in southern Ecuador. This article provides a comparative analysis of both modeling approaches, with emphasis on the evaluation of the model performance. The study revealed that the DBM model better mimics the rainfall-runoff system than the NAM model representing the river basin by a structure composed of three linear reservoirs.
\end{abstract}

Keywords: Hydrological modeling, DBM (Data-Based Mechanistic), Transfer Function (TF), State Dependant Parameters (SDP), Nedbor-Afstromnings Model (NAM), calibration.

\section{INTRODUCCIÓN}

El análisis, la predicción y el pronóstico de caudales es uno de los problemas de mayor interés en la hidrología moderna. Comprender los procesos hidrológicos y tener la capacidad de pronosticarlos a tiempo es esencial para la gestión sostenible de cuencas y de sus recursos naturales, entre los cuales, el agua es quizás el de mayor relevancia dada la diversidad de usos potenciales tales como el doméstico, el industrial, para riego y para generación hidroeléctrica. Esta comprensión es de fundamental importancia si se observa que las actividades humanas producen en la actualidad efectos a escala geológica que se están profundizando en todo el planeta (Sanderson y col., 2002). Así, las manifestaciones más obvias de estos efectos tienen que ver con el abatimiento rápido del almacenamiento en acuíferos debido al bombeo excesivo (Rodell y col., 2009), la distorsión 
significativa de los regímenes de flujo en los ríos debido a la construcción de presas (Poff y col., 2007), la alteración de la recarga de las aguas subterráneas debido a los cambios de uso del suelo (Scanlon y col., 2006), entre muchas otras.

El factor desencadenante de todo esto es la tendencia exageradamente creciente de la población que resulta en un aumento del consumo de energía, agua, comida y espacio de vida, alterando radicalmente nuestro entorno (Hickey y Diaz 1999; Feyen y Vázquez, 2011). Según Palmer y col. (2004) y Wagener y col. (2008), muchos de los servicios de agua dulce se están degradando irremediablemente. La consecuencia acumulada de la creciente demanda y de unos recursos decadentes es la creciente competencia por los recursos naturales, tales como agua, petróleo, minerales, etc. Paralelamente a la disminución de los recursos de agua dulce, la sociedad se enfrenta cada vez más con riesgos de tipo hidrológico, tales como inundaciones y sequías, no sólo en los países en vías de desarrollo, generalmente menos preparados, sino también en los países desarrollados (Feyen y Vázquez, 2011).

Los efectos socioeconómicos y ambientales del exceso y de la escasez de agua son tales que la sociedad pide cada vez más a la comunidad científica y política que desarrolle políticas y planes de gestión adecuados para la prevención y mitigación de los efectos negativos y para la conservación más eficaz del medio ambiente. Esto requiere de predicciones fiables de la ocurrencia, flujo, distribución y calidad del agua bajo escenarios de gestión y de cambio natural/inducido por el hombre.

Los modelos numéricos se utilizan a menudo para llevar a cabo estas predicciones (Vázquez, 2010). Así, los modelos hidrológicos a escala de cuenca están diseñados para estimar escorrentía en base a precipitación, es decir para comprender las interacciones dinámicas entre el clima y la hidrología de la superficie terrestre, a un nivel de detalle según el problema en cuestión (Singh y Woolhiser, 2002). Se emplean en una amplia gama de áreas que van desde la gestión de las cuencas hidrográficas hasta el diseño de ingeniería. Sin embargo, este empleo se da en forma diferente según se los utilice para aplicaciones ingenieriles o para aplicaciones científicas (Singh y Woolhiser, 2002; Savenije, 2009). En este último caso, los científicos están por lo general interesados en averiguar en donde están mal los modelos numéricos y en comprender por qué están comportándose de manera errónea. En el contexto ingenieril, el modelo es visto como una herramienta operacional que permite tomar decisiones con cierto nivel de confianza (Feyen y Vázquez, 2011). En cualquier caso, la modelización numérica está llamada a jugar un papel muy importante ya que puede constituirse en una herramienta muy útil de gestión de los recursos hídricos (Refsgaard, 1997; Vázquez, 2003).

Por lo tanto, si un modelo es capaz de reflejar la esencia de cómo funciona una cuenca hidrográfica (para toda la gama de sus estados posibles), entonces puede ser factible extrapolar con cierta confianza, más allá de las condiciones observadas y llegar a predicciones y/o pronósticos confiables (Refsgaard, 1997; Vázquez, 2003). Esto dependerá del empleo de un protocolo de modelización adecuado (Loague y Green, 1991; Refsgaard, 1997; Vázquez, 2003) que incluya entre otros aspectos: una gran comprensión de los ciclos hidrológicos y bio-geo-químicos y su interacción con los factores de estrés, tanto humanos como naturales; una selección y/o desarrollo de un modelo numérico apropiado; y la aplicación de procesos de calibración, evaluación, análisis de sensibilidad y de incertidumbre (Vázquez y col., 2009).

En cuanto a la selección de una herramienta de modelización adecuada, se debe tener en consideración que un modelo hidrológico de cuencas es un conjunto de descripciones matemáticas de los componentes del ciclo hidrológico. La estructura del modelo y su arquitectura se determinan por el objetivo para el cual se necesita el modelo. Por ejemplo, un modelo hidrológico para el control de inundaciones es bastante diferente de aquel que se utiliza para la operación de embalses, etc. (Singh y Woolhiser, 2002). En este contexto, existen diversos tipos de modelos hidrológicos matemáticos, que varían, entre otros aspectos, en función del tiempo, del espacio y de la aleatoriedad (Chow y col., 1988; Singh y Woolhiser, 2002), así como de otros aspectos más estructurales que tiene que ver con la forma en la que se conceptualizan los distintos componentes y las dinámicas de flujo del ciclo hidrológico (Refsgaard, 1997; Vázquez, 2003). Así, las herramientas pueden ser espacialmente distribuidas o agregadas; basadas en conceptos físicos o en conceptos más empíricos (incluyendo cajas negras); de simulación de eventos o de simulación continua; de minado de datos o de representación de procesos hidrológicos; etc. (Refsgaard y Knudsen, 1996; Refsgaard, 1997; DHI, 2000; Vázquez, 2003; Young, 2011; Young, 2013). 
En este mismo contexto, los modelos pueden ser (i) deterministas, que incluyen ecuaciones diferenciales formuladas siguiendo las leyes físicas y químicas de los procesos descritos y que implican una relación causa/efecto entre los parámetros del modelo y las soluciones de las ecuaciones diferenciales; (ii) conceptuales, que implican representaciones matemáticas simplificadas de los complejos procesos físicos, utilizando pocos parámetros claves; y (iii) de minado de datos, que tienen la capacidad de aprender a partir de los datos.

Un ejemplo de los modelos conceptuales es el Nedbor-Afstromnings Model (NAM) que cuenta con una estructura basada en reservorios lineales (DHI, 2000). En cuanto a los métodos de minado de datos, una posibilidad es la aplicación de Redes Neuronales Artificiales (ANNs) para modelización hidrológica, debido a que las ANNs tienen la capacidad de aprender de forma recursiva a partir de datos y por ende pueden resultar en ahorros significativos en relación al tiempo requerido para el desarrollo del modelo hidrológico. Las ANNs son particularmente adecuadas para la modelización de sistemas no lineales donde las técnicas tradicionales de estimación de parámetros no son muy convenientes (Lorrai y Sechi, 1995; ASCE, 2000a,b; Govindaraju y Rao, 2000). Sin embargo, presenta ciertas limitaciones cuando se requiere la modelización de la estructura interna de las cuencas, en vista de la falta de contenido físico de su estructura y arquitectura (Hsu y col., 1995). En este contexto, como alternativa a esta deficiencia de contenido físico, se plantea el empleo de sistemas híbridos tales como el Modelo Mecanicista Basado en Datos (DBM) que combina por un lado las características de los modelos métricos que, basados en datos observados, caracterizan la respuesta del sistema modelizado, usando para ello estimación u optimización estadística (Wheater y col., 1993); y, por otro lado, de los modelos conceptuales, cuya estructura se basa en las percepciones y experiencia del modelador (Young, 2001).

El presente estudio se ha llevado a cabo en el contexto del desarrollo de una herramienta de pronóstico de caudales a tiempo real para el sistema de generación hidroeléctrica Paute Integral (Ecuador; Vázquez y col., 2012). Esta herramienta se ha pensado de tal forma que incluya distintas aproximaciones para simular la relación precipitación-escorrentía. En este marco de trabajo, se han integrado (i) un módulo de minería de datos; (ii) uno híbrido; y (iii) un módulo basado en un modelo hidrológico conceptual. Para estos módulos se han considerado respectivamente (i) ANNs, ya que tienen la capacidad de aprender de forma recursiva a partir de datos, a una fracción de tiempo en relación al necesario cuando se emplean descripciones complejas de la estructura interna de las cuencas modelizadas; (ii) DBM, que combina el aprendizaje a partir de los datos con la selección de una estructura con cierto significado físico; y (iii) NAM que permite la simulación de la estructura interna de las cuencas modelizadas, en un tiempo corto, lo cual es bastante deseable para un sistema de alerta temprana, y cuya estructura es relativamente fácil de programar (ya que uno de los objetivos del desarrollo del sistema de alerta temprana implica programar herramientas propias, sin restricciones de licencias de uso).

Así, este estudio se ha focalizado en las pruebas de evaluación de las prestaciones de dos de estos módulos, DBM y NAM, en el ámbito de predicción de caudales en subcuencas alto andinas del Austro Ecuatoriano. Se ha decidido considerar solamente los módulos cuya estructura tiene significado físico significativo asociado a la misma; como resultado, no se incluye en la presente evaluación de prestaciones al módulo de minería de datos (ANNs). En particular, se busca apreciar la aplicabilidad de los DBMs para estimar caudales en subcuencas alto andinas de Ecuador, lo cual es un aspecto innovador del presente manuscrito dado que por lo general los DBMs no se han empleado de manera frecuente por parte de la comunidad científica en el país. Si bien NAM está al momento en un proceso de preparación informática, en este estudio se ha utilizado el prototipo comercial (DHI, 2000).

La metodología de evaluación de las prestaciones de las dos herramientas de predicción de caudales incluye aspectos estadísticos y gráficos, así como la aplicación de las estructuras señaladas a dos zonas de estudio ubicadas en las cuencas del Paute y del Jubones, que varían en términos de tamaño y características físicas, considerando de esta forma dos escalas de modelización distintas. Así, se busca caracterizar las prestaciones de estas dos herramientas en actividades de predicción y pronóstico hidrológico en el contexto de la gestión y conservación de ecosistemas alto andinos y de sus recursos hídricos, de fundamental importancia para el desarrollo del Ecuador (CONELEC, 2009 y 2011). 


\section{MATERIALES}

Las subcuencas hidrográficas estudiadas se localizan en los Andes australes del Ecuador y pertenecen a la parte alta de las cuencas de los ríos Paute y Jubones. La primera zona de estudio Tomebamba en Matadero (Fig. 1), ubicada en la cabecera de la cuenca del Paute y con un área de aproximadamente $1275,4 \mathrm{~km}^{2}$, comprende a su vez tres subcuencas que contribuyen a la estación de aforo Tomebamba en Matadero: Tomebamba (extensión aproximada de 380,4 $\mathrm{km}^{2}$ ), Yanuncay (área aproximada de $418,9 \mathrm{~km}^{2}$ ) y Tarqui (área aproximada de $476,13 \mathrm{~km}^{2}$ ). Aguas abajo de la estación de aforo Tomebamba en Matadero, el río continúa llamándose Tomebamba. Esta zona de estudio es accidentada con valles y llanuras, con un rango de elevación que va desde los 2440 hasta los 4420 metros sobre el nivel del mar (m.s.n.m.). Para la presente modelización, se contó con series diarias de evapotranspiración, caudal y lluvia en el período [1 de julio de 1981 - 27 de marzo de 1985], en el que se ha registrado una lluvia media diaria de $2,39 \mathrm{~mm}$ (aproximadamente $778,7 \mathrm{~mm}$ por año). El caudal medio diario fue del orden de $15,4 \mathrm{~m}^{3} \mathrm{~s}^{-1}$ (aproximadamente $3588,2 \mathrm{~m}^{3}$ por año). La evapotranspiración potencial $\left(\mathrm{ET}_{\mathrm{P}}\right)$ media diaria se estimó en aproximadamente $2,25 \mathrm{~mm}$ (unos 823,1 mm por año).

La segunda zona de estudio comprende la microcuenca alto andina de Zhurucay alto ubicada en la cuenca del Jubones. Ésta tiene un área aproximada de $1,34 \mathrm{~km}^{2}$ y cuenta con una estación hidrométrica a la salida de la misma y una estación meteorológica en su parte central (Fig. 1). La zona en la que la microcuenca está enclavada es accidentada con valles y llanuras, principalmente de formación glaciar, con un rango de elevación que va desde los 2760 hasta los 3960 m.s.n.m. La precipitación en la zona es más o menos uniforme y está entre los 1060 y los $1600 \mathrm{~mm}$ anuales. Para la presente modelización, se contó con series temporales observadas de caudal y lluvia, así como variables meteorológica tales como temperatura para la estimación de la evapotranspiración potencial, en el período [ 1 de marzo de 2007 - 30 de noviembre de 2008]. En este período, se registró una lluvia media diaria de 3,99 mm (aproximadamente $1455 \mathrm{~mm}$ por año). La ET $\mathrm{P}_{\mathrm{P}}$ media diaria se estimó en $0,96 \mathrm{~mm}$ (aproximadamente $350 \mathrm{~mm}$ por año). El caudal medio diario fue de 2,70 $\mathrm{mm}$ (aproximadamente $985 \mathrm{~mm}$ por año).

\section{MÉTODOS}

\subsection{El sistema de modelización DBM}

El modelo DBM es híbrido (Young, 2001). Combina por un lado las características de los modelos métricos que, basados en datos observados, caracterizan la respuesta del sistema modelizado, usando para ello estimación u optimización estadística (Wheater y col., 1993); y, por otro lado, de los modelos conceptuales, cuya estructura se basa en las percepciones y experiencia del modelador. La filosofía de la metodología DBM (Young, 1998; Lees, 2000a; Young, 2001, 2002a; Lin, 2003) se puede representar de forma esquemática como se indica en la Fig. 2, en la que se distinguen dos etapas principales y bien diferenciadas que permiten observar la combinación de los modelos métrico por un lado y conceptual por el otro.

La modelización DBM de precipitación-escorrentía presenta una estructura no lineal (Lees, 2000b; Young, 2001, 2002a), conformada por dos componentes: uno lineal, que describe el comportamiento del hidrograma unitario instantáneo; y el no lineal, que describe la relación entre la precipitación medida y la precipitación efectiva, controlando de esta manera el aporte de la precipitación al hidrograma a través del tiempo. El modelo resultante es una versión simplificada de la función de transferencia con parámetros dependientes de estado (SDP) no lineal cuya estructura es la siguiente:

$$
\mathrm{y}_{\mathrm{t}}=\frac{\mathrm{B}\left(\mathrm{z}^{-1}\right)}{\mathrm{A}\left(\mathrm{z}^{-1}\right)} \mathrm{u}_{\mathrm{t}-\delta}+\xi_{\mathrm{t}}
$$



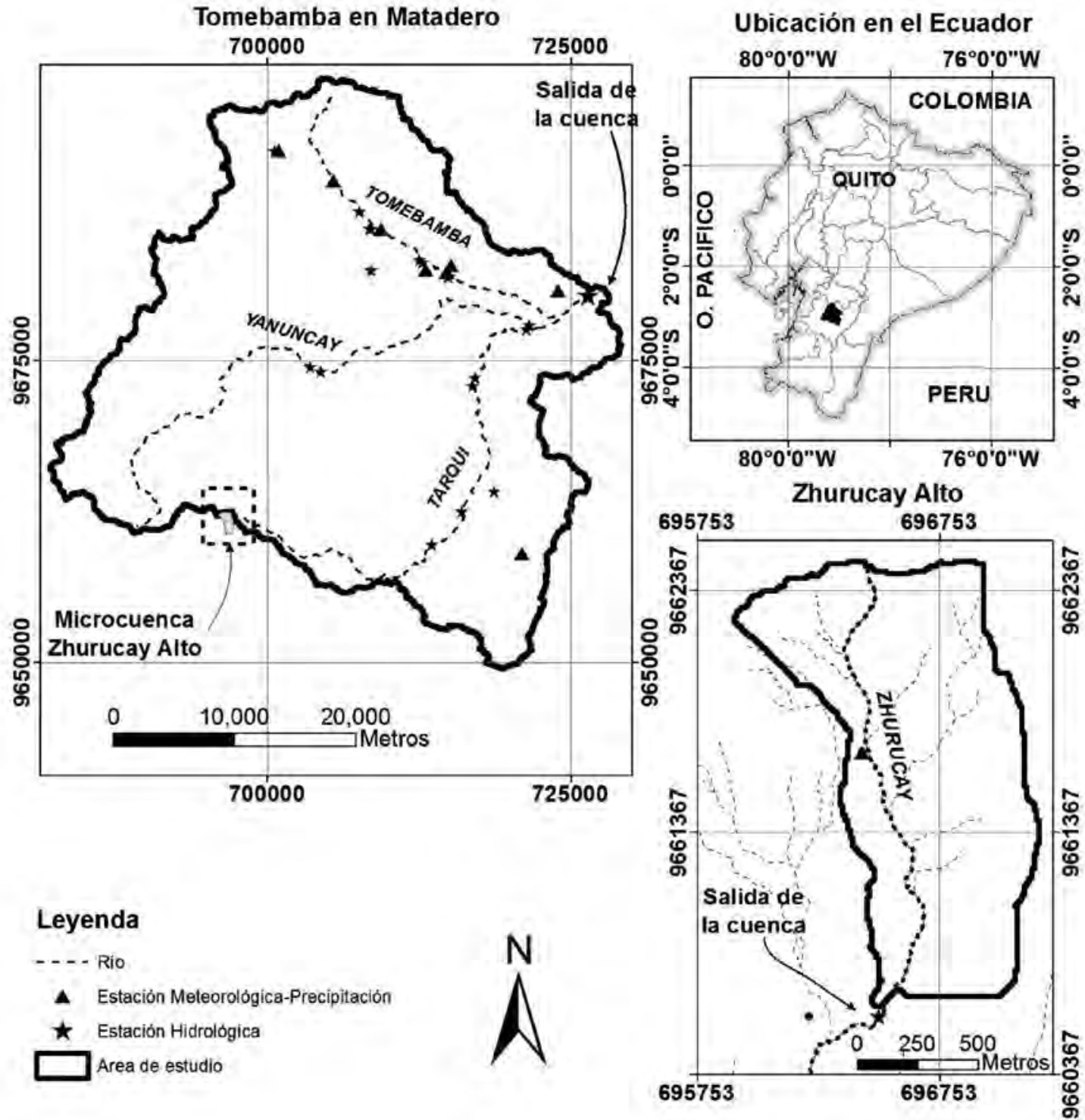

Leyenda
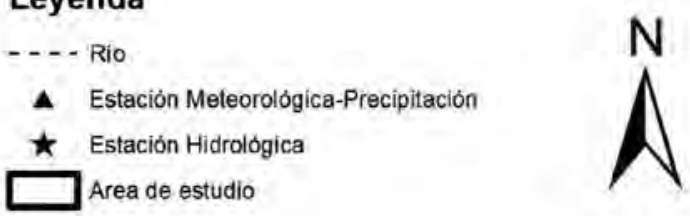

Figura 1. Ubicación de las zonas de estudio y distribución espacial de las respectivas redes hidro-meteorológicas.

donde $\mathrm{y}_{\mathrm{t}}$ es, en el presente caso, el caudal en el tiempo $t$; $\mathrm{u}_{\mathrm{t}-\delta}$, la precipitación efectiva en el tiempo t$\delta . \xi_{\mathrm{t}}$ representa la incertidumbre surgida de la combinación de (i) el ruido de las mediciones; (ii) efectos de otras variables de entrada no medidas; y (iii) el error del modelo. $\mathrm{z}^{-1}$ es un operador de cambio recursivo que se define como $z^{-\mathrm{r}}\left(\mathrm{y}_{\mathrm{t}}\right)=\mathrm{y}_{\mathrm{t}-\mathrm{r}}$ (en el contexto de este artículo $\left.\mathrm{z}^{-\mathrm{r}}\left(\mathrm{y}_{\mathrm{t}}\right)=\mathrm{z}^{-\mathrm{r}}\right), \mathrm{A}\left(\mathrm{z}^{-1}\right)$ y $\mathrm{B}\left(\mathrm{z}^{-1}\right)$ son coeficientes polinomiales constantes de la forma:

$$
\begin{gathered}
\mathrm{A}\left(\mathrm{z}^{-1}\right)=1+\mathrm{a}_{1} \mathrm{z}^{-1}+\mathrm{a}_{2} \mathrm{z}^{-2}+\ldots+\mathrm{a}_{\mathrm{n}} \mathrm{z}^{-\mathrm{n}} \\
\mathrm{B}\left(\mathrm{z}^{-1}\right)=\mathrm{b}_{0}+\mathrm{b}_{1} \mathrm{z}^{-1}+\mathrm{b}_{2} \mathrm{z}^{-2}+\ldots+\mathrm{b}_{\mathrm{m}} \mathrm{z}^{-\mathrm{m}}
\end{gathered}
$$


cuya estructura queda definida por la triada $[\mathrm{n} \mathrm{m} \delta]$ donde, $\mathrm{n}=$ orden de $\mathrm{A}\left(\mathrm{z}^{-1}\right), \mathrm{m}=$ orden de $\mathrm{B}\left(\mathrm{z}^{-1}\right) ; \mathrm{y}$ $\delta$ representa el tiempo de retraso, es decir, el tiempo que una lluvia caída con anterioridad tarda en notarse en el hidrograma observado.

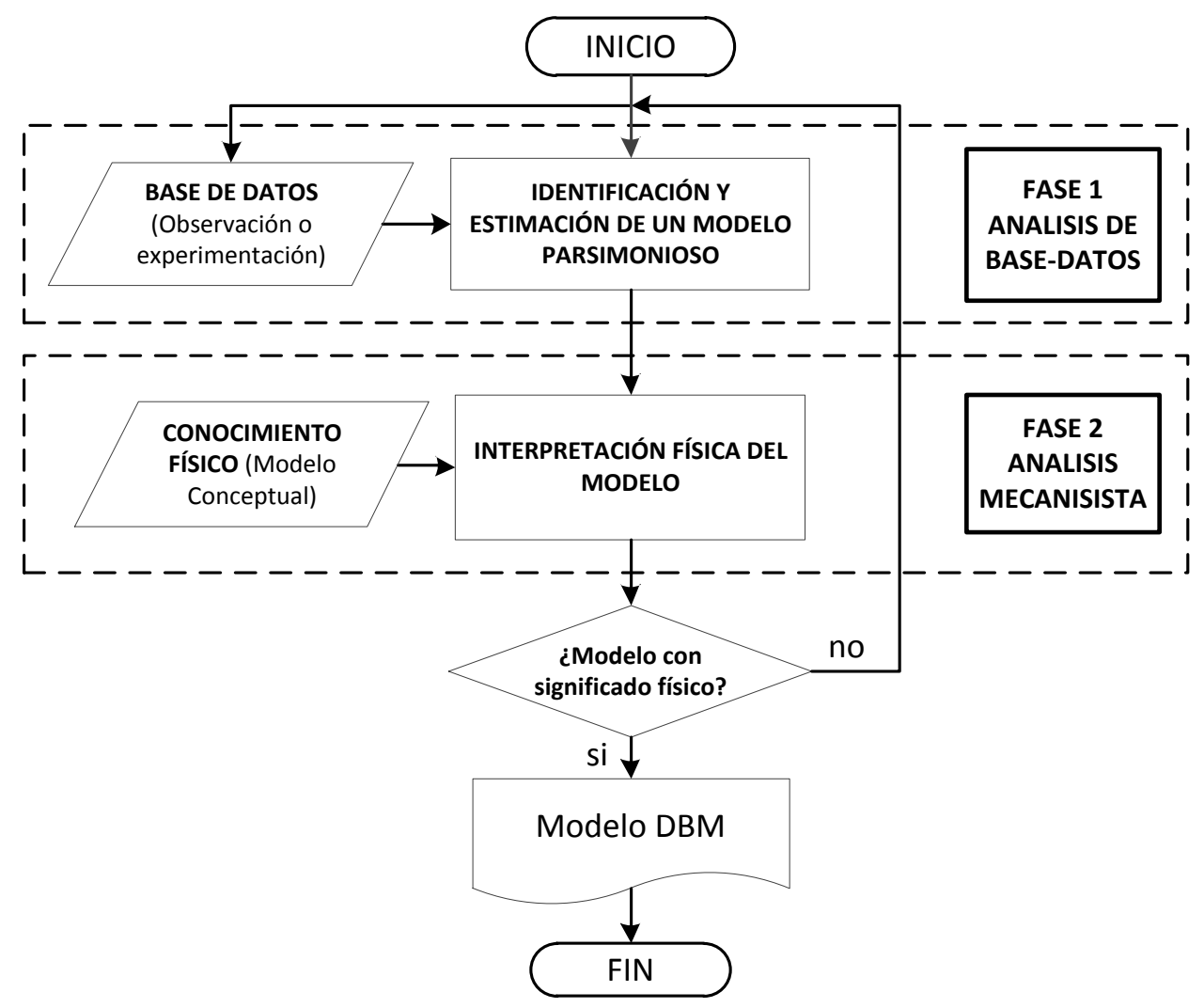

Figura 2. Representación esquemática de la estructura de modelo DBM mediante un diagrama de flujo de procesos.

El componente no lineal del modelo se considera como la precipitación efectiva, que es la causante de las variaciones del caudal observado y en el contexto de la modelización DBM este puede ser cualquier función como $\mathrm{u}_{\mathrm{t}}=\mathrm{f}\left(\mathrm{y}_{\mathrm{t}}, \mathrm{r}_{\mathrm{t}}\right)$ (Young y Tomlin, 2000; Mwakalila y col., 2001; Young, 2001, 2002a, 2002b, 2013). Esto no quiere decir que la precipitación efectiva es una función del caudal, sino mas bien que el caudal está actuando como un sustituto de la capacidad de almacenamiento de la cuenca. Desde el punto de vista hidrológico la variación temporal del almacenamiento de la cuenca sigue el mismo patrón de variación temporal del caudal (Beven, 2004; Lees, 2000a).

Los polinomios $\mathrm{A}\left(\mathrm{z}^{-1}\right)$ y $\mathrm{B}\left(\mathrm{z}^{-1}\right)$ pueden estimarse utilizando cualquier método numérico, uno de ellos es el estimador recursivo de tiempo discreto de entrada múltiple y una única salida (MISO, por sus siglas en inglés) que usa una variable instrumental refinada (RIV; Young y Jakeman, 1979, 1980; Young, 2011; Wang, 2012) utilizada en este estudio y que se encuentra disponible en la herramienta CAPTAIN (Pedregal y col., 2007) para MATLAB®.

Uno de los aspectos más importantes de la modelización DBM es el significado físico que se le puede dar al modelo resultante; de hecho, el modelo no se considera como válido si no tiene una interpretación física adecuada (Fig. 2).

La relación entre la precipitación efectiva y el caudal, que es lineal, dinámica y estocástica, se expresa mediante una función de transferencia (TF, por sus siglas en inglés) lineal discreta cuyo impulso de respuesta representa el hidrograma unitario. Así la TF del modelo puede descomponerse en 
forma paralela de tal manera que se represente por medio de ecuaciones de reservorio lineal de primer orden, dividiendo el aporte de la precipitación efectiva en diversos subflujos en paralelo (aunque no necesariamente reflejen exactamente los subflujos del sistema modelizado), como se muestra en la ecuación siguiente:

$$
y_{t}=x_{1, t}+x_{2, t}+\ldots+x_{k, t}
$$

donde:

$$
\mathrm{x}_{\mathrm{i}, \mathrm{t}}=\frac{\beta_{\mathrm{i}}}{1-\alpha_{\mathrm{i}} \mathrm{z}^{-1}} \mathrm{u}_{\mathrm{t}-\delta}
$$

y $\alpha_{\mathrm{i}}$ y $\beta_{\mathrm{i}}$ son respectivamente las raíces reales de los polinomios $\mathrm{A}\left(\mathrm{z}^{-1}\right)$ y $\mathrm{B}\left(\mathrm{z}^{-1}\right)$ de la Ec. 2 .

Siendo la TF un modelo estocástico, éste tiene la ventaja de que al ser combinado con técnicas de asimilación de datos puede producir múltiples pronósticos a futuro, para lo cual solo basta con convertirle en modelo estado-espacio e implementarlo mediante un filtro de Kalman (Young, 2002b). Esta formulación introduce nuevos parámetros dentro del modelo, denominados hiperparámetros, que toman la forma del radio de varianza del ruido (NVR) capturando de esta manera la naturaleza estocástica de los datos de entrada en las ecuaciones de estado. El modelo resultante puede representarse matemáticamente dentro del filtro de Kalman de la siguiente manera, para predicción (Ec. 5) y corrección (Ec. 6):

$$
\begin{aligned}
& \mathrm{x}_{\mathrm{tt}-1}=\mathrm{Fx}_{\mathrm{t}-1}+\mathrm{Gu}_{\mathrm{t}-\delta} \\
& \mathrm{P}_{\mathrm{tt}-1}=\mathrm{FP}_{\mathrm{t}-1} \mathrm{~F}^{\prime}+\mathrm{Qr} \\
& \mathrm{y}_{\mathrm{t} t-1}=\mathrm{H}^{\mathrm{T}} \mathrm{x}_{\mathrm{tt}-1} \\
& \mathrm{x}_{\mathrm{t}}=\mathrm{x}_{\mathrm{tt}-\mathrm{1}}+\mathrm{P}_{\mathrm{tt}-\mathrm{l}} \mathrm{H}^{\mathrm{T}}\left[1+\mathrm{HP}_{\mathrm{tt}-1} \mathrm{H}^{\mathrm{T}}\right]\left(\mathrm{y}_{\mathrm{t}}-\mathrm{y}_{\mathrm{tt}-1}\right) \\
& \mathrm{P}_{\mathrm{t}}=\mathrm{P}_{\mathrm{tt}-1}+\mathrm{P}_{\mathrm{tt}-1} \mathrm{H}^{\mathrm{T}}\left[1+\mathrm{HP}_{\mathrm{tt} t-1} \mathrm{H}^{\mathrm{T}}\right] \mathrm{HP}_{\mathrm{tt}-1} \\
& \mathrm{y}_{\mathrm{t}}=\mathrm{H}^{\mathrm{T}} \mathrm{x}_{\mathrm{t}}
\end{aligned}
$$

donde,

$$
\mathrm{x}_{\mathrm{t}}=\left[\begin{array}{c}
\mathrm{x}_{1, t} \\
\mathrm{x}_{1, t} \\
\vdots \\
\mathrm{x}_{\mathrm{i}, \mathrm{t}}
\end{array}\right], \mathrm{F}=\left[\begin{array}{cccc}
\alpha_{1} & 0 & \cdots & 0 \\
0 & \alpha_{1} & \cdots & 0 \\
\vdots & \vdots & \ddots & \vdots \\
0 & 0 & \cdots & \alpha_{i}
\end{array}\right], \mathrm{G}=\left[\begin{array}{c}
\beta_{1} \\
\beta_{2} \\
\vdots \\
\beta_{i}
\end{array}\right], H=\left[\begin{array}{c}
1 \\
1 \\
\vdots \\
1
\end{array}\right]
$$

$\mathrm{x}_{\mathrm{t}}$ es el vector estado; $\mathrm{F}$ es la matriz de los parámetros de estado; $\mathrm{G}$ es el vector que contiene los parámetros de la variable de control $\mathrm{u}_{\mathrm{t}}, \mathrm{H}$ es vector observación; $\mathrm{P}$ es la matriz cuadrada de covarianza y $\mathrm{Q}_{\mathrm{r}}$ es una matriz cuadrada que contiene los NVRs y tiene dimensiones similares a la de $\mathrm{F}$ (Kalman, 1960).

Los hiperparámetros pueden estimarse sobre la base de los datos; un método bastante conocido es el de la estimación de máxima verosimilitud (ML) basado en la descomposición del error (Lees, 2000b). La etapa final de la modelización DBM es la validación, proceso mediante el cual se evalúa el potencial de predicción del modelo; ésta se realiza por lo general con un grupo de datos diferente al utilizado en las etapas de identificación y estimación (split-sample; Refsgaard y Knudsen, 1996).

\subsection{El sistema de modelización hidrológica NAM}

El modelo NAM puede definirse como determinista, agregado y conceptual, con requisitos de entrada moderados (Refsgaard y Knudsen, 1996; Vázquez, 2010). El modelo NAM representa los distintos componentes del proceso de precipitación-escorrentía en forma continua mediante el contenido de 
agua en cuatro depósitos diferentes e interrelacionados entre sí (Fig. 3); cada uno de los cuales representa diferentes elementos físicos de almacenamiento de la cuenca (Nielsen y Hansen, 1973; Refsgaard y Knudsen, 1996): la fusión de nieve y los componentes superficial, subsuperficial y subterráneo del hidrograma observado.

La precipitación que es interceptada por la vegetación, y la depositada en la superficie o en las depresiones, contribuye al reservorio superficial. La cantidad de agua del reservorio superficial disminuye constantemente debido al consumo por evaporación así como también por el flujo subsuperficial o flujo interno. Cuando el reservorio superficial excede su capacidad de almacenamiento, el exceso de agua empieza a fluir $\left(\mathrm{P}_{\mathrm{N}}\right)$; parte de este produce escorrentía superficial mientras que la otra parte se infiltra al reservorio de la zona de raíces y al reservorio subterráneo.

El reservorio de la zona baja por su parte representa la capa que se encuentra por debajo de la superficie, en donde las raíces de las plantas aprovechan la humedad del suelo para extraer el agua que se necesita para el proceso de transpiración. El contenido de humedad de este reservorio controla la cantidad de agua que ingresa en el reservorio subterráneo como recarga, además del flujo subsuperficial y el flujo superficial. El contenido de agua en el reservorio de la zona baja está sujeto al consumo por evapotranspiración (ET).

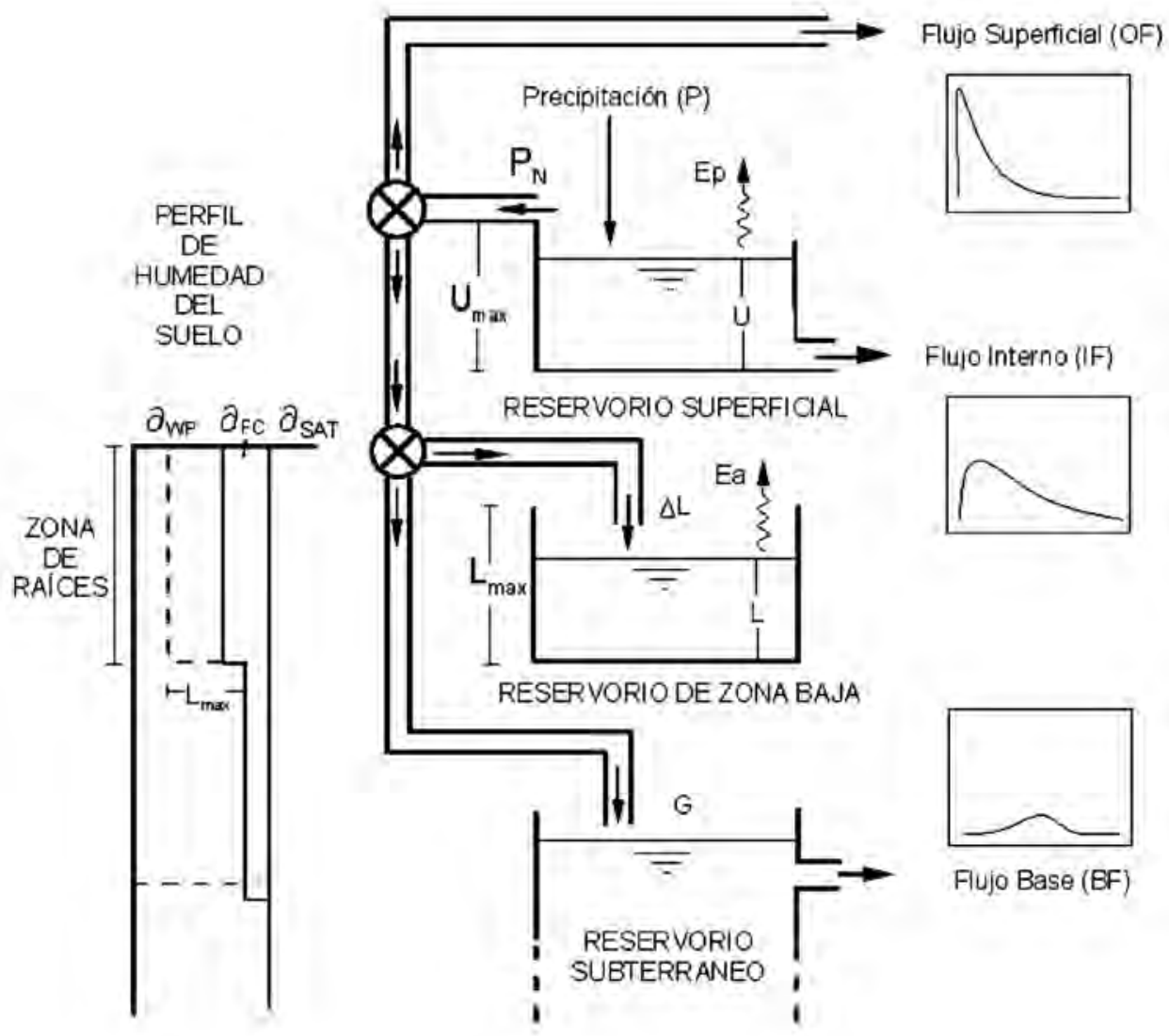

Figura 3. Esquema de la estructura del modelo NAM (en base a DHI, 2000; Nielsen y Hansen, 1973; Vázquez, 2010).

La demanda de ET hasta completar la evapotranspiración potencial $\left(\mathrm{ET}_{\mathrm{P}}\right)$ se obtiene primero del reservorio superficial (Nielsen y Hansen, 1973). Si el contenido de humedad del reservorio superficial es menor que la demanda por evapotranspiración, la fracción restante se obtiene conceptualmente por las raíces de las plantas del reservorio de la zona inferior a una tasa proporcional a la $\mathrm{ET}_{\mathrm{P}} \mathrm{y}$ con una variación lineal en relación al contenido de humedad del suelo. 
Cuando el reservorio superficial supera su máxima capacidad, el exceso de agua empieza a fluir produciendo de esta manera un flujo superficial así como también la infiltración. La parte que contribuye a la escorrentía se define como proporcional al contenido de humedad del suelo y varía linealmente con el mismo. La proporción del exceso de agua que no ingresa a la corriente superficial se infiltra al reservorio de la zona baja. Una fracción de esta aumenta el contenido de humedad del reservorio de la zona baja y la otra parte se filtra hacia las profundidades y recarga el reservorio de agua subterránea.

El flujo interno se asume que es proporcional al contenido de agua del reservorio superficial, con un tiempo de recesión constante y varía linealmente con la humedad relativa del reservorio de la zona baja. El flujo subsuperficial, se transita a través de dos reservorios lineales en serie con un mismo tiempo de recesión, constante. El flujo superficial también está basado en el concepto de reservorio lineal, pero con una constante de tiempo variable. Por último, la cantidad de agua infiltrada al reservorio subterráneo depende del contenido de humedad del reservorio subsuperficial y de su capacidad (DHI, 2000).

El sistema de modelización conceptual NAM está basado en conceptos físicos y ecuaciones semiempíricas. Al ser un modelo agregado, el NAM considera cada cuenca como una sola unidad, por lo tanto, los parámetros y las variables representan valores promedio para toda la cuenca. Como resultado, algunos de los parámetros del modelo se podrían, en principio, evaluar a partir de datos físicos de la cuenca, aunque esto no siempre es factible (Célleri y col., 2003), por lo que la estimación de parámetros finales se debe realizar mediante la calibración del modelo en base a datos hidrológicos observados (Vázquez, 2010).

\subsection{Evaluación del desempeño de los modelos}

Para evaluar el rendimiento de los modelos se establecieron varios criterios con el objetivo principal de percibir cuán bien se ajustan las series de tiempo simuladas a las observadas (Refsgaard y Knudsen, 1996; Beven, 2004). Los mismos comprenden una combinación de análisis visual de gráficas apropiadas (hidrogramas, hidrogramas acumulados, diagramas de dispersión, etc.) y de medidas estadísticas (métricas o índices).

Así, en este estudio se ha considerado el índice de eficiencia $\mathrm{EF}_{2}$ propuesto por (Nash y Sutcliffe, 1970) con un rango de variación que va desde $-\infty$ hasta 1 (valor óptimo); el coeficiente de determinación ponderado $\mathrm{wR}^{2}$ (Krause $y$ col., 2005) con variación entre 0 y 1 (valor óptimo); el índice concordancia de grado j, $\mathrm{d}_{\mathrm{j}}$ (Willmont, 1981; Legates y McCabe, 1999; Vázquez, 2003) con un rango similar al de $w \mathrm{R}^{2}$. Las expresiones matemáticas de estos índices son:

$$
\begin{aligned}
& \mathrm{EF}_{2}=1-\frac{\sum_{\mathrm{i}=1}^{\mathrm{n}}\left(\mathrm{O}_{\mathrm{i}}-\mathrm{P}\right)_{\mathrm{i}}^{2}}{\sum_{\mathrm{i}=1}^{\mathrm{n}}\left(\mathrm{O}_{\mathrm{i}}-\overline{\mathrm{O}}\right)^{2}}
\end{aligned}
$$

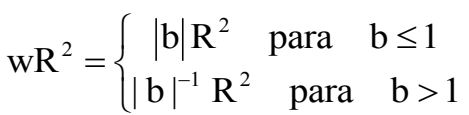

$$
\begin{aligned}
& d_{j}=1-\frac{\sum_{i=1}^{n} O_{i}-\left.P_{i}\right|^{j}}{\sum_{i=1}^{n}\left(\left|P_{i}-\bar{O}\right|+\left|O_{i}-\bar{O}\right|\right)^{j}}
\end{aligned}
$$

donde, 


$$
\mathrm{R}^{2}=\left(\frac{\sum_{\mathrm{i}=1}^{\mathrm{n}}\left(\mathrm{O}_{\mathrm{i}}-\overline{\mathrm{O}}\right)\left(\mathrm{O}_{\mathrm{i}}-\overline{\mathrm{P}}\right)}{\sqrt{\sum_{\mathrm{i}=1}^{\mathrm{n}}\left(\mathrm{O}_{\mathrm{i}}-\overline{\mathrm{O}}\right)^{2}} \sqrt{\sum_{\mathrm{i}=1}^{\mathrm{n}}\left(\mathrm{P}_{\mathrm{i}}-\overline{\mathrm{P}}\right)^{2}}}\right)^{2}
$$

$\mathrm{O}_{\mathrm{i}}$ es la observación i, $\mathrm{P}_{\mathrm{i}}$ es la simulación i y $\mathrm{O}$ es el valor medio de las observaciones en el período de evaluación.

El índice $\mathrm{R}^{2}$ solo cuantifica la correlación de las series observada y simulada sin tomar en cuenta apropiadamente la coincidencia entre las mismas (Legates y McCabe, 1999); adicionalmente es demasiado sensible a la simulación de valores extremos máximos (Legates y McCabe, 1999; Vázquez y col., 2002). Por estos motivos no es muy aconsejada su utilización en modelización hidrológica; en su lugar se suele usar $\mathrm{EF}_{2}$ que se enfoca más en la coincidencia entre las series observada y simulada y es menos sensible a valores extremos máximos (Legates y McCabe, 1999; Vázquez y col., 2002). Se supone que $d_{j}$ con $j=1$ es aún menos sensible a la simulación de eventos extremos máximos (Legates y McCabe, 1999; Vázquez, 2003; Krause y col., 2005). En este mismo contexto, (Krause y col., 2005) sugieren utilizar el gradiente $\mathrm{b}$ de la regresión en la cual está basado $\mathrm{R}^{2}$ para mejorar significativamente las prestaciones del índice $\mathrm{R}^{2}$ a niveles comparables al índice $\mathrm{EF}_{2}$. Aunque los tres índices considerados son estimaciones del error medio total (error combinado sistemático y aleatorio; Vázquez y col., 2008), y por ende no aportan información distinta en relación a la medición del error de simulación numérica, se han considerado en este estudio para facilitar la comparación con otros estudios similares publicados en la literatura científica y para explorar el comportamiento de los índices $d_{j} y w R^{2}$.

\subsection{Parametrización, calibración y validación de los modelos}

Para los procesos de identificación, calibración y validación del modelo se utilizó un ensayo splitsample (Refsgaard y Knudsen, 1996). De esta forma, y en función de la disponibilidad de datos (precipitación, escorrentía y $\mathrm{ET}_{\mathrm{P}}$ ), para la subcuenca del Tomebamba en Matadero, se definieron los siguientes intervalos: [1 de julio de 1981 - 3 de octubre de 1983] para identificación, y calibración; y [4 de octubre de 1983 - 27 de marzo de 1985] para validación. De la misma manera, para la subcuenca del Zhurucay, se definieron los intervalos [27 de octubre de 2006 - 17 de mayo de 2008] para identificación, y calibración y [17 de mayo de 2008 - 05 de julio de 2009] para validación.

En el caso de la modelización DBM se utilizó como variables de entrada las mediciones de precipitación y escorrentía. La elección de la estructura más adecuada del modelo en el caso de la metodología DBM se basó en la evaluación de las distintas estructuras ensayadas y en particular mediante la función SRIV para la TF lineal y la función SDP para la parte no lineal. Estas funciones conjuntamente con otras funciones estadísticas utilizadas en el presente estudio se encuentran disponibles en la herramienta CAPTAIN.

Para el caso de la modelización NAM, las variables de entrada son la precipitación y la evapotranspiración y para la calibración del modelo se necesita las mediciones de escorrentía. Los parámetros del modelo que se han calibrado en este estudio se listan en la Tabla 2 conjuntamente con sus rangos de variación definidos en base a DHI (2000) y Vázquez (2010). El modelo para las dos zonas de estudio, se calibró mediante un proceso automático disponible en el sistema de modelización hidrológica empleado (DHI, 2000).

Adicionalmente, NAM necesita como condiciones iniciales los niveles iniciales de los diferentes reservorios y los caudales iniciales superficial, subsuperficial y subterráneo. En el presente estudio se consideró un período de calentamiento (warming up period) de tres meses para atenuar los efectos de las condiciones iniciales sobre la calidad de las predicciones (Vázquez, 2010). 


\section{RESULTADOS Y DISCUSIÓN}

En cuanto a la identificación de la estructura DBM más adecuada para la modelización de la subcuenca del Tomebamba en Matadero, el estudio mostró que la estructura [2 3 0] es la más efectiva paramétricamente (estructura parsimoniosa), lo cual coincide con (Young, 2002b; Romanowicz y col., 2008), quienes sugieren que, si se dispone de una base de datos diaria de precipitación-caudal los modelos, deberían ser generalmente de tercer orden, implicando así que el comportamiento del sistema precipitación-caudal se representaría de mejor manera mediante un sistema de tres reservorios lineales con tiempos de respuesta diferentes (coincidiendo de cierta forma con la representación considerada en NAM).

La estructura escogida en el presente estudio implica que el tiempo que transcurre desde que la precipitación se observa hasta que el caudal se registra en la estación de aforo es menor a un día (lo cual se puede observar también en la Fig. 4). Para la estimación de la parte no-lineal de la estructura DBM se utilizó una función potencial de la forma $\mathrm{f}\left(\mathrm{y}_{\mathrm{t}}\right)=\mathrm{c} y_{t}^{\beta}$, la misma que se optimizó empleando el método Simplex (Nelder y Mead, 1965). El proceso de optimización de los parámetros de la función potencial arrojó los valores $\mathrm{c}=1,33$ y $\beta=0,97$.

Tabla 1. Rangos de variación de los valores de los parámetros del modelo NAM incluidos en el presente análisis de calibración.

\begin{tabular}{|c|c|c|c|c|c|c|}
\hline \multirow{2}{*}{ Parámetro } & \multirow{2}{*}{ Descripción } & \multirow{2}{*}{ Unidad } & \multicolumn{2}{|c|}{$\begin{array}{l}\text { Intervalo de } \\
\text { variación }\end{array}$} & \multicolumn{2}{|c|}{$\begin{array}{l}\text { Valor calibrado } \\
\text { (automáticamente) }\end{array}$} \\
\hline & & & Mínimo & Máximo & $\begin{array}{l}\text { Tomebamba- } \\
\text { Matadero }\end{array}$ & $\begin{array}{l}\text { Zhurucay } \\
\text { alto }\end{array}$ \\
\hline $\mathrm{U}_{\max }$ & $\begin{array}{l}\text { Capacidad máxima del reservorio } \\
\text { superficial. }\end{array}$ & {$[\mathrm{mm}]$} & 5,0 & 35,0 & 10,6 & 20,0 \\
\hline $\mathrm{L}_{\max }$ & $\begin{array}{l}\text { Capacidad máxima del reservorio } \\
\text { subsuperficial. }\end{array}$ & {$[\mathrm{mm}]$} & 50,0 & 400,0 & 120,5 & 100,0 \\
\hline $\mathrm{CQ}_{\mathrm{OF}}$ & $\begin{array}{l}\text { Coeficiente de escorrentía del } \\
\text { flujo superficial. }\end{array}$ & {$[-]$} & 0,00 & 1,00 & 0,97 & 0,73 \\
\hline $\mathrm{CK}_{\mathrm{IF}}$ & $\begin{array}{l}\text { Constante de tiempo para el flujo } \\
\text { interno. }\end{array}$ & [hora] & 200,0 & 2000,0 & 223,6 & 335,09 \\
\hline $\mathrm{CK}_{12}$ & $\begin{array}{l}\text { Constante de tiempo de recesión } \\
\text { de flujo subsuperficial. }\end{array}$ & [hora] & 3,0 & 72,0 & 37,6 & 18,06 \\
\hline $\mathrm{T}_{\mathrm{OF}}$ & $\begin{array}{l}\text { Umbral para el flujo } \\
\text { subsuperficial. }\end{array}$ & {$[-]$} & 0,00 & 0,90 & 0,15 & 0,01 \\
\hline $\mathrm{T}_{\mathrm{IF}}$ & Umbral para el flujo superficial. & {$[-]$} & 0,00 & 0,90 & 0,06 & 0,01 \\
\hline $\mathrm{T}_{\mathrm{G}}$ & $\begin{array}{l}\text { Umbral para la recarga del } \\
\text { acuífero. }\end{array}$ & {$[-]$} & 0,00 & 0,90 & 0,03 & 0,19 \\
\hline $\mathrm{CK}_{\mathrm{BF}}$ & $\begin{array}{l}\text { Constante de tiempo de recesión } \\
\text { del flujo base. }\end{array}$ & [hora] & 500,0 & 5000,0 & 1814,1 & 3888,5 \\
\hline
\end{tabular}

Los parámetros obtenidos para la TF de la estructura seleccionada para Matadero se muestran en la siguiente ecuación:

$$
y_{t}=\frac{0,113(0,0018)-0,016(0,0016) z^{-1}-0,089(0,0001) z^{-2}}{1-1,454(0,0004) z^{-1}+0,464(0,0002) z^{-2}} u_{t-\partial}
$$

donde, la varianza de los parámetros estimados es el factor que se encuentra entre paréntesis. Esta ecuación (11) descompuesta, presenta la estructura DBM para la cuenca del Tomebamba en Matadero de la siguiente manera: 


$$
y_{t}=0,113 u_{t-\partial}+\frac{0,982}{1-0,009 z^{-1}} u_{t-\partial}+\frac{0,472}{1-0,140 z^{-1}} u_{t-\partial}
$$

Esta estructura se representa gráficamente en la Fig. 4; los detalles de la descomposición, por ejemplo del tiempo de residencia (T) y de la fracción de volumen de aporte (d) de cada componente se presentan en la Tabla 2. De la misma manera, para la microcuenca del Zhurucay, el estudio mostró que la estructura $\left[\begin{array}{lll}2 & 2 & 0\end{array}\right]$ es la más efectiva, implicando así que el comportamiento del sistema precipitación-caudal se representaría de mejor manera para esta microcuenca mediante un sistema de dos reservorios lineales con tiempos de respuesta distintos. En cuanto al tiempo de retraso, este presentó una condición parecida a la de la cuenca anterior, a una escala subdiaria.

Para la estimación de la parte no-lineal de la estructura DBM también se utilizó una función potencial de la forma $\mathrm{f}\left(\mathrm{y}_{\mathrm{t}}\right)=\mathrm{c} y_{t}^{\beta}$, cuya optimización arrojó los valores $\mathrm{c}=0,03$ y $\beta=0,37$. Los parámetros obtenidos para la TF de la estructura seleccionada se muestran en la siguiente ecuación:

$$
y_{t}=\frac{0,346(0,0008)-0,337(0,0001) z^{-1}}{1-1,424(0,0004) z^{-1}+0,434(0,0001) z^{-2}} u_{t-\partial}
$$

que descompuesta, presenta la siguiente estructura:

$$
y_{t}=\frac{0,983}{1-0,007 z^{-1}} u_{t-\partial}+\frac{0,441}{1-0,339 z^{-1}} u_{t-\partial}
$$

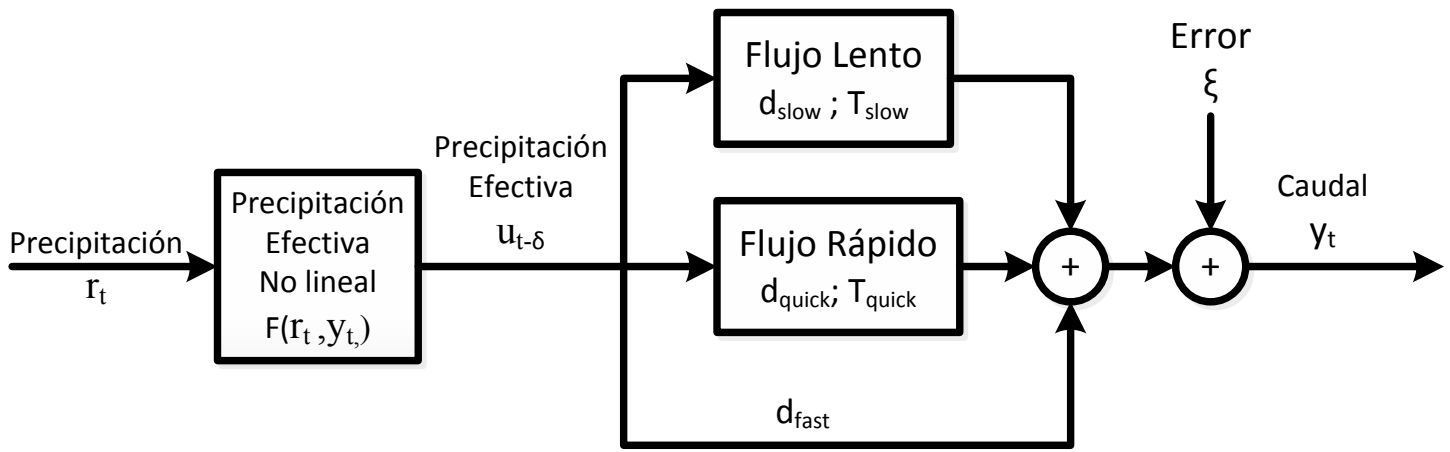

Figura 4. Esquema de la descomposición de la lluvia efectiva considerada en la estructura del modelo DBM.

El tiempo de residencia y la fracción del volumen de aporte de cada componente del flujo se listan en la Tabla 2.

Las ecuaciones $11,12,13$ y 14 puede interpretarse de la siguiente manera: para la cuenca del Tomebamba en Matadero la precipitación efectiva $u_{t}$ aporta al caudal con tres sub-flujos diferentes: uno muy rápido (instantáneo) que representa un $12 \%$ del caudal total y que se debe probablemente a procesos superficiales; un componente de flujo rápido con un tiempo de residencia de 1,33 días, que aporta un 30\% al hidrograma y que probablemente se debe a procesos de poca profundidad; y un flujo lento con un tiempo de residencia de 58,32 días que aporta un $58 \%$ al cauce total del río, siendo además su componente principal, el mismo que se asocia a procesos subsuperficiales y subterráneos.

Para la microcuenca de Zhurucay alto $\mathrm{u}_{\mathrm{t}}$ aporta al hidrograma con dos subflujos diferentes: uno rápido con un tiempo de residencia de 1,22 días y que aporta un 1,5\% al caudal del río; y un segundo componente de flujo lento con un tiempo de residencia de 58,36 días que aporta un 98,5\% al cauce total del río. Para esta microcuenca, tanto la estructura de la TF como los porcentajes de contribución 
de cada reservorio apuntalan las observaciones de campo y los resultados de simulaciones anteriores que apuntan hacia una contribución significativa del flujo subsuperficial proveniente del reservorio subsuperficial (suelos), lo cual sugeriría una alta dependencia del hidrograma observado en la hidrología subsuperficial. Sin embargo, queda aún por trabajar más adelante en la interpretación de información y el análisis de los aportes de los diferentes subsistemas hidrológicos al hidrograma total de la microcuenca Zhurucay alto.

Tabla 2. Descomposición paralela de la función de transferencia (modelo DBM) en función de la zona de estudio.

\begin{tabular}{|c|c|c|c|}
\hline Flujo & T (día) & $\begin{array}{c}\text { Fracción del } \\
\text { volumen total, d (\%) }\end{array}$ & SS \\
\hline \multicolumn{4}{|c|}{ Tomebamba en Matadero } \\
\hline Lento & 58,32 & 58 & 0,51 \\
\hline Rápido & 1,33 & 30 & 0,26 \\
\hline Instantáneo & $<1$ & 12 & 0,11 \\
\hline \multicolumn{4}{|c|}{ Microcuenca Zhurucay alto } \\
\hline Flujo lento & 58,36 & 98,5 & 0,236 \\
\hline Flujo rápido & 1,22 & 1,5 & 0,003 \\
\hline
\end{tabular}

Leyenda: $\mathrm{T}=$ Tiempo de residencia; $\mathrm{SS}=$ Steady State

Tabla 3. Evaluación cuantitativa de la calidad de las predicciones de los modelos DBM y NAM.

\begin{tabular}{|c|c|c|c|c|c|c|c|c|c|}
\hline \multirow{2}{*}{ Parámetro } & \multirow{2}{*}{ Unidad } & \multicolumn{4}{|c|}{ Calibración } & \multicolumn{4}{|c|}{ Validación } \\
\hline & & Qobs & DBM TF & DBM KF & NAM & Qobs & $\overline{\text { DBM TF }}$ & DBM KF & NAM \\
\hline \multicolumn{10}{|c|}{ Tomebamba en Matadero } \\
\hline \multirow[t]{2}{*}{ Qmed } & {$[\mathrm{mm}]$} & 13,85 & 13,57 & 13,84 & 13,84 & 17,80 & 17,77 & 17,43 & 16,63 \\
\hline & {$[\%]$} & & 97,98 & 99,93 & 99,93 & & 99,83 & 97,92 & 93,43 \\
\hline \multirow[t]{2}{*}{ Qacum } & {$[\mathrm{mm}]$} & 11355,8 & 11126,6 & 11346,9 & 11352,3 & 9717,1 & 9704,9 & 9514,1 & 9079,4 \\
\hline & {$[\%]$} & -- & 97,98 & 99,92 & 99,97 & -- & 99,87 & 97,91 & 93,44 \\
\hline $\mathrm{EF}_{2}$ & {$[-]$} & -- & 0,81 & 0,81 & 0,71 & -- & 0,75 & 0,83 & 0,66 \\
\hline$w R^{2}$ & {$[-]$} & -- & 0,79 & 0,81 & 0,52 & -- & 0,80 & 0,77 & 0,38 \\
\hline$d_{1}$ & {$[-]$} & -- & 0,85 & 0,86 & 0,73 & -- & 0,84 & 0,86 & 0,73 \\
\hline \multicolumn{10}{|c|}{ Microcuenca de Zhurucay alto } \\
\hline \multirow[t]{2}{*}{ Qmed } & {$\left[\mathrm{m}^{3} \mathrm{~s}^{-1}\right]$} & 0,03 & 0,03 & 0,03 & 0,04 & 0,05 & 0,05 & 0,05 & 0,04 \\
\hline & {$[\%]$} & -- & 99,91 & 99,24 & 113,43 & -- & 99,97 & 99,81 & 86,16 \\
\hline \multirow[t]{2}{*}{ Qacum } & {$\left[\mathrm{m}^{3} \mathrm{~s}^{-1}\right]$} & 19,5 & 19,5 & 19,4 & 22,2 & 19,4 & 19,4 & 19,4 & 16,7 \\
\hline & {$[\%]$} & -- & 99,91 & 99,24 & 113,43 & -- & 99,97 & 99,81 & 86,16 \\
\hline $\mathrm{EF}_{2}$ & {$[-]$} & -- & 0,84 & 0,93 & 0,88 & -- & 0,72 & 0,88 & 0,58 \\
\hline$w R^{2}$ & {$[-]$} & -- & 0,80 & 0,87 & 0,80 & -- & 0,70 & 0,80 & 0,31 \\
\hline$d_{1}$ & {$[-]$} & -- & 0,85 & 0,89 & 0,82 & -- & 0,80 & 0,86 & 0,67 \\
\hline
\end{tabular}

Leyenda: Qmed = caudal medio (diario); Qacum = caudal acumulado; Qobs = caudal observado; KF $=$ Filtro de Kalman.

Finalmente, la modelización DBM consiste en expresar a la TF descompuesta en un sistema estado-espacio e incorporarla dentro de un filtro de Kalman, mejorando de esta manera el pronóstico y su interpretación física. Los parámetros principales de comparación cuantitativa entre las simulaciones sin (DBM TF) y con (DBM KF) aplicación del filtro de Kalman se presentan en la Tabla 3. En cuanto 
a la calibración del modelo NAM, aplicado a las zonas de estudio, la Tabla 1 lista los valores optimizados automáticamente, tal como se señaló anteriormente.

Con el modelo DBM se obtienen mejores predicciones del balance hídrico con respecto al modelo NAM, tal como se ilustra en la Tabla 3 que lista los valores de los estadísticos de evaluación de la bondad de ajuste de las dos herramientas de predicción hidrológica en los períodos de calibración y validación (split-sample). En este estudio se hicieron uso además de otras herramientas gráficas de evaluación de las predicciones de los modelos. Así, en la Fig. 5 se presenta hidrogramas (período de calibración) y los diagramas de dispersión (período de validación) de las series de tiempo simuladas y observadas para la cuenca del Tomebamba en Matadero. Ambos modelos parecen ajustarse favorablemente a los datos observados con una ventaja a favor del DBM en sus dos variantes (sin el uso del filtro de Kalman y con el uso del filtro, respectivamente).
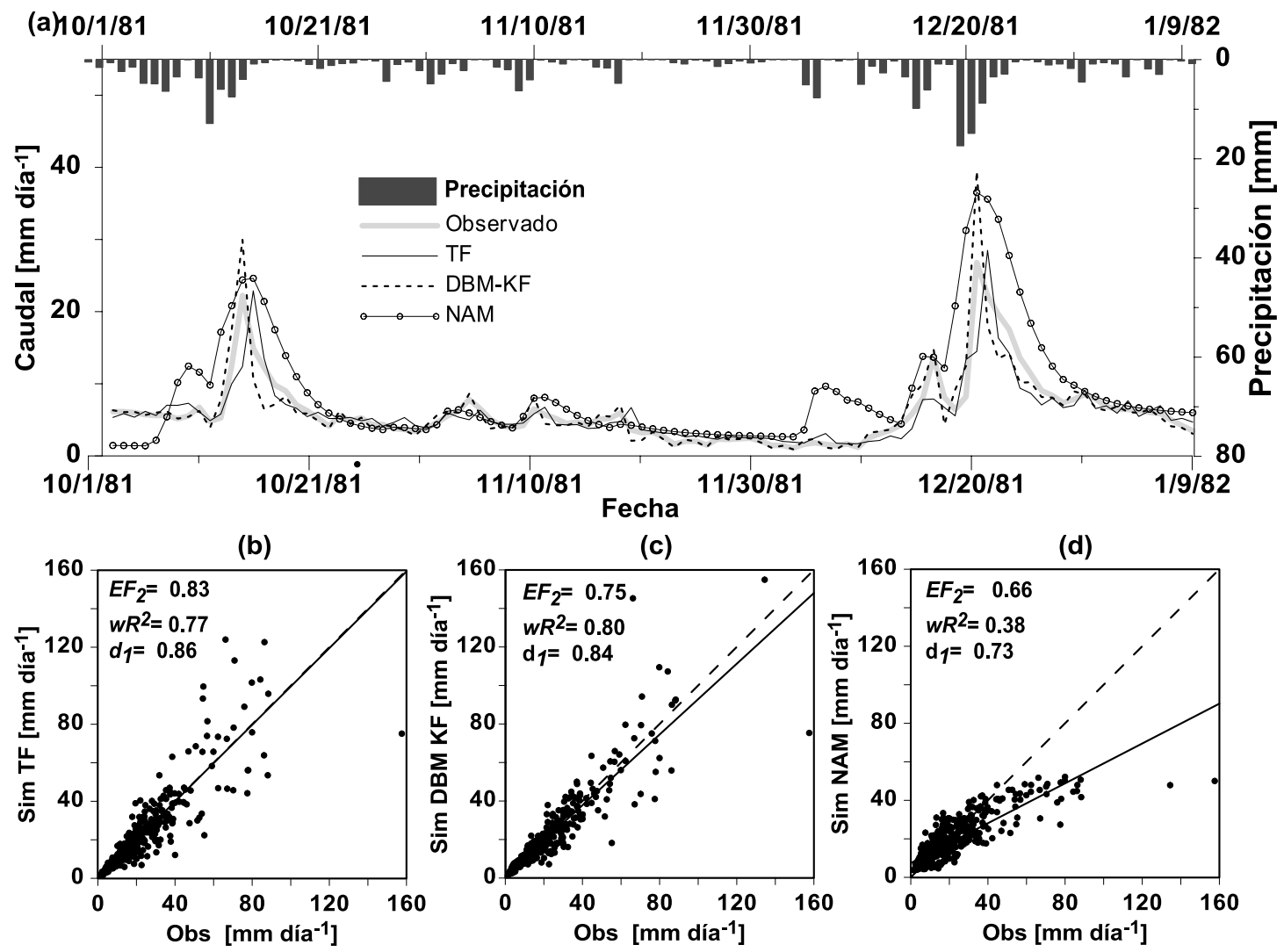

Figura 5. Predicciones para la cuenca del Tomebamba en Matadero: a) hidrograma de caudales observado y simulado (calibración); (b), (c) y (d) diagramas de dispersión de valores observados y simulados validación. Los diagramas de dispersión incluyen las líneas 1:1 (entrecortada) y de tendencia lineal de los datos (continua).

Considerando tanto los resultados estadísticos como los gráficos es claro que, de manera global, con respecto al NAM, el DBM presenta un mejor rendimiento tanto en el período de calibración como en el de validación, con una reducción general de la calidad de las predicciones en el período de validación, lo cual es muy normal en el ámbito de la modelización hidrológica.

En el DBM, la implementación del filtro de Kalman para las dos zonas de estudio mejora el rendimiento en la simulación, tanto de los caudales picos como de los bajos. Por otro lado, existe una evidente subestimación de caudales con el modelo NAM (Figs. 5 y 6). Adicionalmente, es importante notar como el caudal medio diario de las zonas de estudio (Tabla 3) difiere significativamente en los 
períodos de calibración y validación, lo cual sugiere un potencial cambio del régimen de caudales en función del tiempo, que justificaría el bajo rendimiento en los períodos de validación, de una manera global parecería que NAM es mucho más sensible a esos cambios.

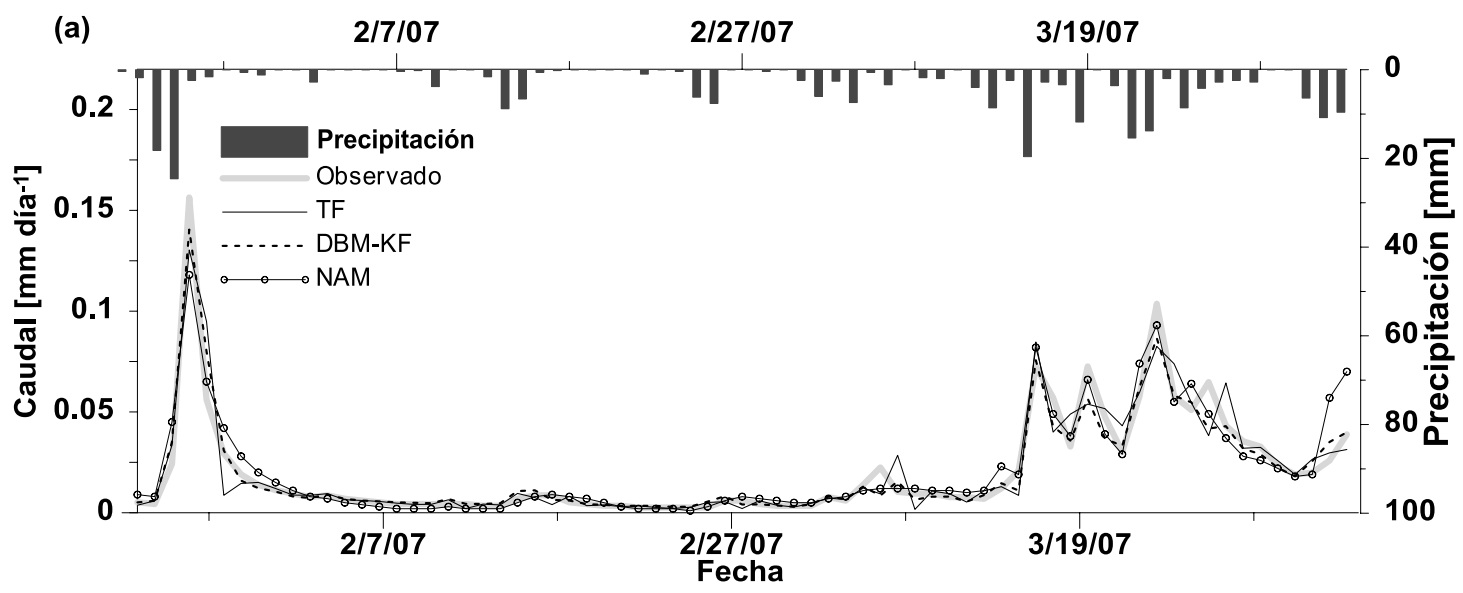

(b)

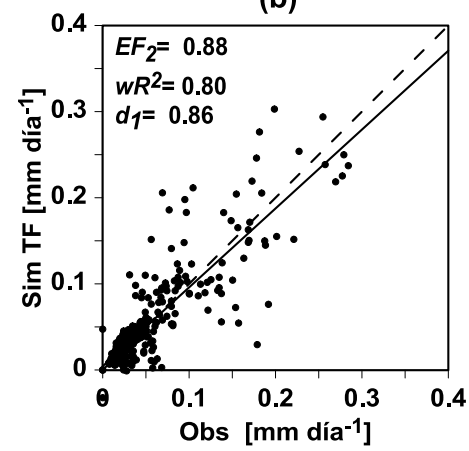

(c)

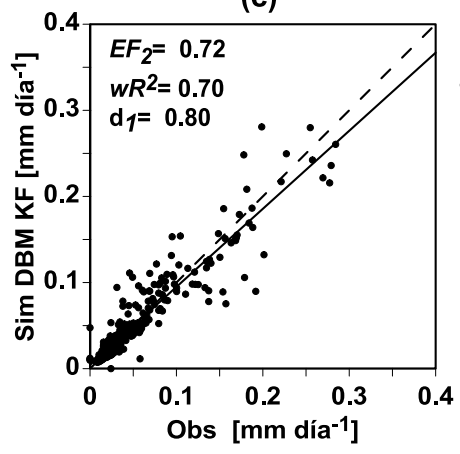

(d)

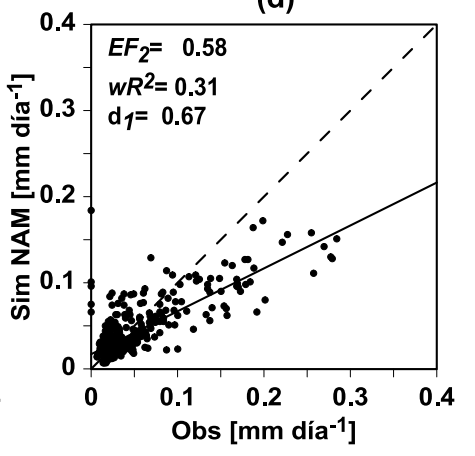

Figura 6. Predicción para la microcuenca de Zhurucay alto: a) hidrograma de caudales observados y simulados (calibración); (b), (c) y (d) diagramas de dispersión de valores observados y simulados (validación). Los diagramas de dispersión incluyen las líneas 1:1 (entrecortada) y de tendencia lineal de los datos (continua).

\section{CONCLUSIONES Y TRABAJO FUTURO}

Los sistemas de modelización DBM y NAM se han sometido a un proceso de evaluación de su capacidad de predicción considerando datos de dos subcuencas alto andinas. Por un lado, NAM (DHI, 2000) representa un modelo hidrológico conceptual y por otro lado el DBM (Young y col., 2001) es un modelo que combina características de un modelo conceptual con un modelo black-box de minado de datos.

Las predicciones obtenidas con el modelo DBM exhiben una superioridad con respecto a las predicciones del modelo NAM, lo cual sugiere que la estructura DBM, por sus características intrínsecas, es capaz de adaptarse a los datos de las cuencas de estudio permitiendo inclusive una interpretación física en función de la descomposición de la TF. La estructura del modelo NAM, aparentemente, se ve afectada por una sobre-sensibilidad a los datos de $\mathrm{ET}_{\mathrm{P}}$ (Vázquez, 2010), produciendo predicciones bastante desfavorables en relación a las conseguidas con la estructura DBM (con y sin filtro de Kalman). 
Sin embargo, a pesar de que los resultados obtenidos están de acuerdo con el objetivo principal del presente trabajo, se debe considerar que los mismos están influenciados por condiciones exógenas al sistema de modelización como pueden ser la disponibilidad de datos y su calidad, etc. Por lo tanto, para llegar a una conclusión general se requieren todavía de varias pruebas de validación, semejantes a las propuestas en el presente artículo.

Así, el presente estudio reveló que el DBM mostró en general un rendimiento aceptable con los registros disponibles para el presente estudio; se ajustó aceptablemente al caudal observado en el período de calibración sin reducir severamente su rendimiento durante el período de validación. En principio, esto alienta a pensar que si se dispone de un período adecuado de registros de unos pocos años, el sistema de modelización DBM se podría utilizar como una herramienta confiable para diferentes análisis tales como el de rellenar vacíos en los registros observados. Además, debido a la utilización de algoritmos avanzados para la calibración del modelo, el modelo DBM sería en principio más fácil de calibrar que NAM, ya que existen menos parámetros y se requiere menos experiencia del modelador, contrastando con el sistema de modelización NAM cuya utilización demanda mayor experiencia hidrológica, inclusive cuando se emplea el módulo de calibración automática disponible en el sistema de modelización (DHI, 2000).

En cuanto al conjunto de herramientas gráficas y estadísticas empleadas para la evaluación de las predicciones de los modelos, el presente estudio confirmó la validez de los métodos gráficos. El estudio reveló además que los tres índices en efecto reflejan de forma semejante la bondad de ajuste entre series observadas y las simuladas, tal como se esperaba en vista de que son una medida semejante del error medio sistemático y del aleatorio (en particular $\mathrm{wR}^{2} \mathrm{y} \mathrm{EF}_{2}$ ). Esto ilustra que $\mathrm{wR}^{2}$ (Krause $y$ col., 2005), variación del estadístico $\mathrm{R}^{2}$ (Legates y McCabe, 1999; Vázquez y col., 2002), tiene un comportamiento mucho más adecuado que éste; en este estudio, incluso este estadístico es más sensible que $\mathrm{EF}_{2}$ al momento de percibir la menor calidad de las predicciones asociadas al modelo NAM (DHI, 2000). Por su parte, $d_{1}$ también exhibió una sensibilidad adecuada para caracterizar la calidad de las simulaciones. En todo caso, queda todavía por examinar detalladamente si $\mathrm{d}_{1}$ es un estadístico que no se ve afectado por las simulaciones de eventos extremos, como si acontece con $\mathrm{EF}_{2}$ $\mathrm{y} w \mathrm{R}^{2}$ que se basan en al cuadrado de las diferencias entre las predicciones y las simulaciones.

\section{AGRADECIMIENTOS}

El presente artículo se desarrolló en el contexto de la tesis de grado del primer autor (Ingeniería Civil, UC), y de los proyectos dirigidos por el segundo autor: (i) Establecimiento de modelos numéricos para casos selectos de la gestión de recursos hídricos, financiado por la UC; (ii) Gestión de datos y modelización hidrológica para Soporte al Pronóstico de Alerta Temprana del Sistema Paute Integral, financiado por la Corporación Eléctrica del Ecuador (CELEC E.P.) y la UC; y (iii) Cuantificación hidrológica de los componentes del paisaje, financiado por la UC.

\section{REFERENCIAS}

ASCE, 2000a. Artificial neural networks in hydrology. 1: Preliminary concepts. J. Hydrol. Eng., 5(2), $115-123$.

ASCE, 2000b. Artificial neural networks in hydrology. 2: Hydrology applications. J. Hydrol. Eng., 5(2), 124-137.

Beven, K.J., 2004. Rainfall - Runoff Modelling: The Primer. Wiley, 253 pp.

Célleri, R., L. Timbe, R. Vázquez, 2003. Assessment of the relation between the NAM rainfall-runoff model parameters and the physical catchment properties. HIP-VI UNESCO Technical Documents in Hydrology, 66, 9-16. 
Chow, V.T., D.R. Maidment, L.W. Mays, 1988. Applied Hydrology. McGraw-Hill International Editions, Singapore, 572 pp.

CONELEC, 2009. Estadística del sector eléctrico ecuatoriano. Consejo Nacional de Electricidad, Quito, Ecuador, 388 pp.

CONELEC, 2011. Estadística del sector eléctrico ecuatoriano. Consejo Nacional de Electricidad, Quito, Ecuador, 189 pp.

DHI, 2000. MIKE 11 A Modelling System for Rivers and Channels. Danish Hydraulic Institute, Denmark, $292 \mathrm{pp}$.

Feyen, J., R.F. Vázquez, 2011. Modeling hydrological consequences of climate and land use change Progress and Challenges. Maskana, 2(2), 83-100.

Govindaraju, R.S., A.R. Rao, 2000. Artificial Neural Networks in hydrology. Springer-Verlag, Boston, USA, 348 pp.

Hickey, J.T., G.E. Diaz, 1999. From flow to fish to dollars: An integrated approach to water allocation. J. Am. Water Resour. Assoc., 35(5), 1053-1067.

Hsu, K. L., H.V. Gupta, S. Sorooshian, 1995. Artificial neural network modeling of the rainfall-runoff process. Water Resour. Res., 31(10), 2517-2531.

Kalman, R.E., 1960. A New Approach to Linear Filtering and Prediction Problems. Trans. ASME -J. Basic Engin., 82, 35-45.

Krause, P., D.P. Boyle, F. Bäse, 2005. Comparison of different efficiency criteria for hydrological model assessment. Adv. Geosci., 5, 89-97.

Lees, M.J., 2000a. Data-based mechanistic modelling and forecasting of hydrological systems. $J$. Hydroinformatics, 2(1), 15-34.

Lees, M.J., 2000b. Advances in Transfer Function Based Flood Forecasting. In: Flood forecasting: What does current research offer the practitioner? Lees, M.J., P. Walsh (eds.), BHS occasional paper No. 12, Centre for Ecology and Hydrology, British Hydrological Society, 7, 40-54.

Legates, D.R., G.J. McCabe, 1999. Evaluating the use of goodness-of-fit Measures in hydrologic and hydroclimatic model validation. Water Resour. Res., 35, 233-241.

Lin, Z., 2003. Modeling environmental systems under uncertainty: Towards a synthesis of data-based and theory-based models (Ph.D. Thesis). University of Georgia, Georgia, USA, 209 pp.

Loague, K., R.E. Green, 1991. Statistical and graphical methods for evaluating solute transport models: overview and applications. J. Contam. Hydrol., 7: 51-73.

Lorrai, M., G.M. Sechi, 1995. Neural nets for modeling rainfall runoff transformations. Water Resour. Manage., 9, 299-313.

Mwakalila, S., P. Campling, J. Feyen, G. Wyseure, K. Beven, 2001. Application of a data-based mechanistic modelling (DBM) approach for predicting runoff generation in semi-arid regions. Hydrol. Process., 15, 2281-2295.

Nash, I.E., I.V. Sutcliffe, 1970. River flow forecasting through conceptual models. J. Hydrol., 10, 282290.

Nelder, J.A., R. Mead, 1965. A Simplex Method for Function Minimization. Comput. J., 7, 308-313.

Nielsen, S.A., E. Hansen, 1973. Numerical simulation of the rainfall-runoff process on a daily basis. Nordic Hydrol., 4, 171-190.

Palmer, M., E. Bernhardt, E. Chornesky, S. Collins, A. Dobson, C. Duke, B. Gold, R. Jacobson, S. Kingsland, R. Kranz, M. Mappin, M.L. Martinez, F. Micheli, J. Morse, M. Pace, M. Pascual, S. Palumbi, O.J. Reichman, A. Simons, A. Townsend, M. Turner, 2004. Ecology for a crowded planet. Science, 304, 1251-1252.

Pedregal, D.J., C.J. Taylor, P.C. Young, 2007. System identification, time-series analysis and forecasting. CAPTAIN handbook: The Captain Toolbox. Downloaded from http://captaintoolbox.co.uk/Captain_Toolbox.html, 273 pp.

Poff, N.L., J.D. Olden, D. Merritt, D. Pepin, 2007. Homogenization of regional river dynamics by dams and global biodiversity implications. Proc. Natl. Acad. Sci.USA, 104, 5732-5737. 
Refsgaard, J.C., 1997. Parameterisation, calibration and validation of distributed hydrological models. J. Hydrol., 198, 69-97.

Refsgaard, J.C., Knudsen, J., 1996. Operational validation and intercomparison of different types of hydrological models. Water Resour. Res., 32, 2189-2202.

Rodell, M., I. Velicogna, J.S. Famiglietti, 2009. Satellite-based estimates of groundwater depletion in India. Nature, 460, 999-1002.

Romanowicz, R.J., P.C. Young, K.J. Beven, F. Pappenberger, 2008. A data based mechanistic approach to nonlinear flood routing and adaptive flood level forecasting. Adv. Water Resour., 31, 1048-1056.

Sanderson, E.W., M. Jaitech, M.A. Levy, K.H. Redford, A.V. Wannebo, G. Woolmer, 2002. The human footprint and the last of the wild. BioScience, 52, 891-904.

Savenije, H.H.G., 2009. The art of hydrology. Hydrol. Earth Syst. Sci., 13, 157-161.

Singh, V.P., D.A. Woolhiser, 2002. Mathematical Modeling of Watershed Hydrology. J. Hydrol. Eng., 7(4), 270-292.

Scanlon, B.R., K.E. Keese, A.L. Flint, L.E. Flint, C.B. Gaye, M. Edmunds, I. Simmers, 2006. Global synthesis of groundwater recharge in semiarid and arid regions. Hydrol. Process., 20, 3335-3370.

Vázquez, R.F., 2003. Assessment of the performance of physically based distributed codes simulating medium size hydrologycal systems. PhD Thesis, Faculty of Engineering, ISBN 90-5682-416-3, KU Leuven, Leuven, Bélgica, 335 pp.

Vázquez, R.F., 2010. Modelación hidrológica de una microcuenca Altoandina ubicada en el Austro Ecuatoriano. Maskana, 1, 79-90.

Vázquez, R.F., L. Feyen, J. Feyen, J.C. Refsgaard, 2002. Effect of grid size on effective parameters and model performance of the MIKE-SHE code. Hydrol. Process., 16, 355-372.

Vázquez, R.F., P. Willems, J. Feyen, 2008. Improving the predictions of a MIKE SHE catchmentscale application by using a multi-criteria approach. Hydrol. Process., 22, 2159-2179.

Vázquez, R.F., K. Beven, J. Feyen, 2009. GLUE based assessment on the overall predictions of a MIKE SHE application. Water Resour. Manag., 23(7), 1325-1349.

Vázquez, R.F., R. Célleri, E. Samaniego, P. Vanegas, L.V. Campozano, J.M. Orellana, A.O. Vázquez, 2012. Proyecto Gestión de datos y Modelización Hidrológica para soporte al pronóstico de alerta temprana del Sistema Paute Integral (Informe Final). Corporación Eléctrica del Ecuador (CELEC E.P.), Cuenca, Ecuador.

Wagener, T., M. Sivapalan, B.L. McGlynn, 2008. Catchment classification and services. Toward a new paradigm for catchment hydrology driven by societal needs. In: Anderson, M.G. (ed.), Encyclopedia of Hydrological Sciences, John Wiley, Chichester, UK.

Wang, L., 2012. System identification, environmental modelling and control system design. SpringerVerlag, London, UK, 653 pp.

Wheater, H.S., A.J. Jakeman, K.J. Beven, 1993. Progress and directions in rainfall-runoff modelling. In: Jakeman, A.J., M.B. Beck, M.J. McAleer (eds.), Modelling change in environmental systems John Wiley, Chichester, UK, 101-132.

Willmont, J.C., 1981. On the validation of models. Physic. Geogr., 2, 184-194.

Young, P., Jakeman, A., 1979. Refined instrumental variable methods of recursive time-series analysis Part I. Single input, single output systems. Int. J. Control, 29, 1-30.

Young, P., Jakeman, A., 1980. Refined instrumental variable methods of recursive time-series analysis Part III. Extensions. Int. J. Control, 31, 741-764.

Young, P.C., 1973. Recursive approaches to time-series analysis. Bulletin Inst. Maths and its Applications, 10, 209-224.

Young, P.C., 1993. Time variable and state dependent modelling of non-stationary and non-linear time series. In: Rao, T.S. (ed.), Development in Time Series Analysis. Chapman \& Hall, London, UK, 374-413. 
Young, P.C., 1998. Data-based mechanistic modelling of environmental, ecological, economic and engineering systems. Environ. Modell. Softw., 13, 1867-1874.

Young, P.C., 2001. Data-Based mechanistic modelling and validation of rainfall-flow processes. In: Anderson, M.G., P.D. Bates (eds.), Model Validation: Perspectives in Hydrological Science. John Wiley, Chichester, UK, 117-161.

Young, P.C., 2002a. Data-based mechanistic and top-down modelling. Proc. Int. Conf. on Integrated Assessment and Decision Support (iEMSs2002), 24-27.

Young, P.C., 2002b. Advances in real-time flood forecasting. Phil. Trans. R. Soc. Lond. A, 360, 14331450.

Young, P.C., 2011. Recursive estimation and time-series analysis: An introduction for the student and practitioner. ( $2^{\text {nd }}$ ed.), Springer-Verlag, $505 \mathrm{pp}$.

Young, P.C., 2013. Hypothetico-inductive data-based mechanistic modeling of hydrological systems. Water Resour. Res., 1-67. Downloaded from http://captaintoolbox.co.uk/Captain_Toolbox.html.

Young, P.C., P. McKenna, J. Bruun, 2001. Identification of non-linear stochastic systems by state dependent parameter estimation. Int. J. Control, 74, 1837-1857.

Young, P.C., C.M. Tomlin, 2000. Data-Based mechanistic modelling and adaptive flow forecasting. In: Lees, M.J., P. Walsh (eds.), What does current research offer the practitioner? BHS occasional paper No 12, Centre for Ecology and Hydrology, British Hydrological Society, UK, 25-39. 